\title{
Late-time Spectral Observations of Type IIP Supernovae
}

\author{
Jeffrey M. Silverman ${ }^{1}$, Stephanie Pickett ${ }^{1}$, J. Craig Wheeler ${ }^{1}$ and \\ Alexei V. Filippenko ${ }^{2}$ \\ ${ }^{1}$ University of Texas at Austin, \\ Austin, TX, United States \\ email: jsilverman@astro.as .utexas.edu \\ ${ }^{2}$ University of California - Berkeley, \\ Berkeley, CA, United States
}

Keywords. supernovae: general, techniques: spectroscopic

We are analysing late-time (older than about $150 \mathrm{~d}$ past explosion) optical spectra of Type II-Plateau (IIP) supernovae (SNe), which are H-rich SNe that come from red supergiant (RSG) progenitors. The dataset includes nearly 100 spectra of about 40 objects, making this the largest sample of SN IIP nebular spectra ever investigated. Quantitative criteria from within the spectra themselves are employed to determine if an observation is truly nebular, and thus should be included in the study. We present the temporal evolution of the fluxes, shapes, and velocities of various emission lines (see, for example, Fig. 1). These measured values are also compared to photometric data in order to search for correlations that can allow us to gain insight into the diversity of RSG progenitors and learn more about the details of the explosion itself.

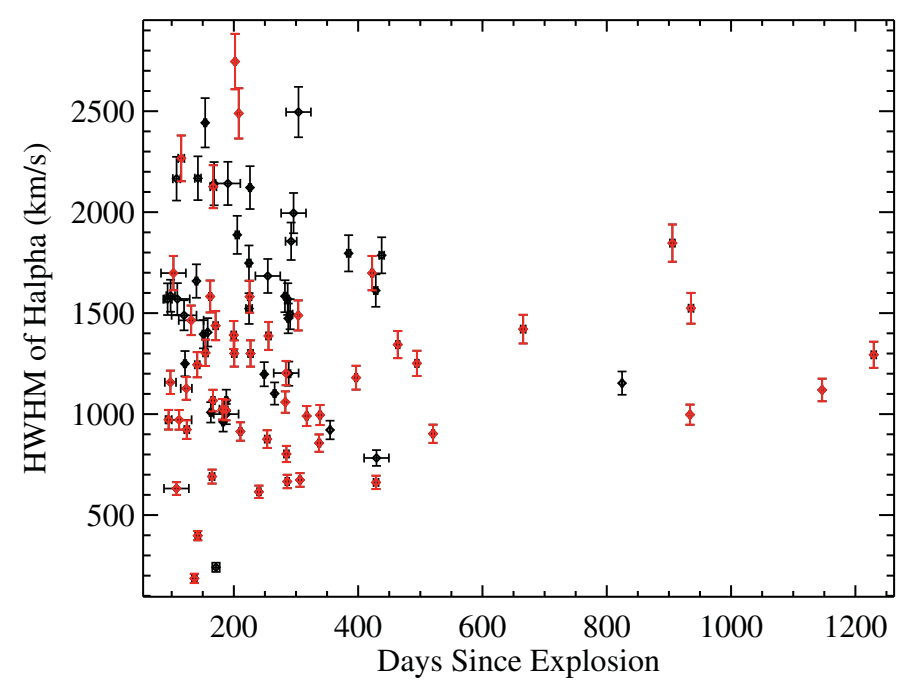

Figure 1. The half-width at half maximum (HWHM) of $\mathrm{H} \alpha$ emission versus time. Red points have (plateau) $M_{V}>-16.5 \mathrm{mag}$, while black points have $V$-band plateau magnitudes brighter than -16.5 mag. A Kolmogorov-Smirnov test indicates that the HWHM is significantly lower for the fainter objects as compared to the brighter ones. 